\title{
Article \\ Probabilistic Inference with Interval Probabilities
}

\author{
Oleg Uzhga-Rebrov ${ }^{1 *}$ and Galina Kuleshova ${ }^{2}$ \\ 1 Rezekne Academy of Technologies, Rezekne, Latvia; rebrovs@tvnet.lv \\ 2 Riga Technical University, Riga, Latvia; galina.kulesova@rtu.lv \\ * Correspondence: rebrovs@tvnet.lv
}

\begin{abstract}
Probabilistic inference problems have very broad practical applications. To solve this kind of problems under conditions of certainty, an effective mathematical apparatus has been developed. In real situations, obtaining deterministic estimates of relevant probabilities is often difficult; therefore, problems with handling uncertain estimates of probabilities appear. This paper examines the problem of probabilistic inference with probability trees provided that the initial probabilities are given in the form of intervals of their possible values.
\end{abstract}

Keywords: consistent interval probabilities; generalized probability intervals; interval probabilities; Kaucher arithmetic; permissible interval probabilities; probabilistic inference;probability trees; reachable interval probabilities

\section{Introduction}

In many practical problems, it is often necessary to determine the probabilities of events under consideration based on the probabilities of other events. Such problems are called probabilistic inference problems. Elementary tasks of probabilistic inference include [1,2].

1. marginalisation of joint probabilities;

2. conditioning of probabilities;

3. calculation of the posterior probabilities based on the joint probabilities;

4. calculation of the posterior probabilities using Bayes' formula.

More complicated are problems of probabilistic inference for probability trees and belief networks. More details about methods of solving this kind of tasks can be found in [1, 2].

Effective techniques are developed for solving probabilistic inference problems for the cases when initial probability estimates are uncertain, namely, when the estimates are given in the interval or fuzzy form.

This paper examines the problem of probabilistic inference under the condition that initial values of relevant probabilities are set as intervals of their possible values.

\section{Basic Concepts and Definitions of Interval Probabilities}

Let there exist a set of random events $A=\left\{a_{i}, i=1, \ldots, n\right\}$. Let us assume that probabilities of these events are assigned not in the deterministic form but in the form of intervals of possible values of these probabilities

$$
\left[l_{i}, u_{i}\right], \quad i=1, \ldots n
$$

where $l_{i}$ - lower (the least) possible value of probability $p_{i}=p\left(a_{i}\right)$; 
$u_{i}$ - upper (the largest) possible value of probability $p_{i}=p\left(a_{i}\right)$.

It is evident that by selecting randomly or systematically by one value of $p_{i} \in\left[l_{i}, u_{i}\right]$, $i=1, \ldots, n$, we will obtain a set of deterministic probability estimates $\left\{p_{i}, i=1, \ldots, n\right\}$. In literature, such sets of deterministic values of probabilities are often treated as probability distributions.

In [3], a set of all possible such probabilities are formally defined as follows:

$$
\mathbf{P}=\left\{p_{i} \in p(A) / l_{i} \leq p_{i} \leq u_{i}, \forall i\right\},
$$

where $p(A)$ denotes a set of all possible probability estimates defined in the set of random events A.

To avoid a situation when $\mathbf{P}=\varnothing$, boundary values of probability intervals have to satisfy these limiting conditions:

$$
\sum_{i=1}^{n} l_{i} \leq 1 \leq \sum_{i=1}^{n} u_{i}
$$

Probability intervals satisfying conditions (2), in [3] are called proper intervals. It is evident that in tasks of interval probabilistic inference one should always operate with proper intervals only.

In general case, $p_{i} \geq l_{i}$ and $p_{i} \leq u_{i}, \forall p_{i} \in A$. If

$$
l_{i}=\inf _{p_{i} \in \mathbf{P}} \text { and } u_{i}=\sup _{p_{i} \in \mathbf{P}}, \forall i,
$$

It means that deterministic probability values can be selected over the entire interval $\left[l_{i}, u_{i}\right]$, including its boundaries. In [3], probability intervals meeting conditions (3) are called reachable intervals.In [3], it is proven that for reachable probability intervals these inequalities are valid:

$$
\begin{aligned}
& \sum_{j \neq i} l_{j}+u_{i} \leq 1, \forall i ; \\
& \sum_{j \neq i} u_{j}+l_{i} \geq 1, \forall i .
\end{aligned}
$$

Calculations of relevant interval probabilities are made according to rules of interval arithmetic, as well as by some special expressions. The use of such special expressions is stipulated by the need to ensure reachable intervals of resulting probabilities. For illustration, the calculation of the posterior probabilities by Bayes' formula can be mentioned given that the initial probabilities are set in the interval form. Several methods for extending classical Bayes' formula to interval probabilities are known; one method was proposed in [4 - 7]. The essence of this method is as follows. Let $F=(\Omega, A, l(\cdot))-F$-field and $\mathbf{C}$ - division $\Omega$, such that $(F, \mathbf{C})$ is a luminary constellation. Then the following information enables reconstruction of the initial field $F$ :

1. $F_{C}=\left(C, A(C), l_{C}(\cdot / C)\right)-$ marginal $F$-field regarding the division $C:$ "prior probabilities".

2. $F_{C}=\left\{C, A(C), l_{C}(\cdot / C) / C \in \mathbf{C}\right\}-$ a set of fields of conditional $F$ - probability according to the canonical concept. 
An F - field of complete probabilities can be calculated based on the assigned conditional probabilities $\left[l_{C}(\cdot / C), u_{C}(\cdot / C)\right]$ and probabilities of division $p(C)$. A complete interval probability of event $A \in C$ is calculated by expression

$$
\mathrm{p}(\mathrm{A})=[1(\mathrm{~A} / \mathrm{C}) 1(\mathrm{C}), \mathrm{u}(\mathrm{A} / \mathrm{C}) \mathrm{u}(\mathrm{C})]
$$

For each $B \in A$, intuitive concept of conditional probability creates an $F$-field

$$
\operatorname{ip}(A / B)=\left[\inf _{\text {pìM }} p(A / B), \sup _{\text {pîM }} p(A / B)\right] \text {. }
$$

Conditional probabilities $i p(A / B)$ are the desired posterior probabilities.

Although the application of the proposed method produces correct results, it will not be used in this paper due to the complexity and difficulties in interpretation of the results obtained. Instead, a method proposed in [8,9] will be used.

The method under consideration is based on the concept of generalized intervals. Classical interval is identified as a set of real numbers, whereas a generalized interval is identified with the help of predicates that are filled with real numbers; its boundaries are not ordered in common sense. A generalized interval $x=[\underline{x}, \bar{x}] \in K R$ is called proper if $\underline{x} \leq \bar{x}$, and improper, if $\underline{x} \geq \bar{x}$. A set of proper intervals is denoted as $I R=\{[\underline{x}, \bar{x}] / \underline{x} \leq \bar{x}\}$, but a set of improper intervals is denoted as $\overline{I R}=\{[\underline{x}, \bar{x}], \underline{x} \geq \bar{x}\}$. Operations on the generalized intervals are defined based on Kaucher arithmetic [10]. In the set of generalized intervals, these specific mathematical operations are defined.

$$
\operatorname{prox}=[\min (\underline{\mathrm{x}}, \overline{\mathrm{x}}), \max (\underline{\mathrm{x}}, \overline{\mathrm{x}})]
$$

Operation [7] results in a proper generalized interval.

$$
\operatorname{impx}=[\max (\underline{x}, \bar{x}), \min (\underline{x}, \bar{x})] .
$$

The result of operation (8) is an improper generalised interval.

The operation that follows transforms a proper generalized interval into the improper generalized interval.

$$
\text { dual } \mathbf{x}=[\bar{x}, \underline{x}] .
$$

In [8], author proposes this interval version of Bayes' formula:

$$
p\left(E_{i} / A\right)=\frac{p\left(A / E_{i}\right) p\left(E_{i}\right)}{\sum_{j=1}^{n} \operatorname{dualp}\left(A / E_{j}\right) \operatorname{dualp}\left(E_{j}\right)},
$$

where $E_{i}, \quad i=1, \ldots, n$, - are mutually separate event separations in $\Omega$ and $\sum_{j=1}^{n} p\left(E_{j}\right)=1, d u a l(\cdot)$ is defined in (9).

Boundary values of intervals of the posterior probabilities $p\left(E_{i} / A\right)$ can be calculated using this expression [8]: 


$$
\left[1\left(E_{i} / A\right), u\left(E_{i} / A\right)\right]=\left[\frac{1\left(A / E_{i}\right) 1\left(E_{i}\right)}{\sum_{j=1}^{n} 1\left(A / E_{j}\right) 1\left(E_{j}\right)}, \frac{u\left(A / E_{i}\right) u\left(E_{i}\right)}{\sum_{j=1}^{n} u\left(A / E_{j}\right) u\left(E_{j}\right)}\right] .
$$

It is necessary to understand that according to common expression (10), interval values of probabilities in both denominators in expression (11) are inverted values of initial interval values of probabilities; for example, if $p\left(E_{j}\right)=\left[1\left(E_{j}\right), u\left(E_{j}\right)\right]$, then $\operatorname{dualp}\left(E_{j}\right)=\left[u\left(E_{j}\right), 1\left(E_{j}\right)\right]$.

When we have only two relevant events $E$ and $E^{c}$, boundary values of the posterior conditional probabilities can be calculated by these expressions [8]:

$$
\begin{aligned}
& 1(E / A)=\frac{1(A / E) 1(E)}{1(A / E) 1(E)+1\left(A / E^{c}\right) 1\left(E^{c}\right)} \\
& u(E / A)=\frac{u(A / E) u(E)}{u(A / E) u(E)+u\left(A / E^{c}\right) u\left(E^{c}\right)} .
\end{aligned}
$$

It should be taken into consideration that in the denominators of expressions $(12, a, b)$ not the initial boundary values of probabilities $p(\cdot)$, but their inverted values $d u a l(\cdot)$ need to be used. To simplify calculations, all necessary calculations can be made using the initial interval values of probabilities, and the result can then be inverted by expression

$$
\sum_{j=1}^{n} \operatorname{dualp}\left(A / E_{j}\right) \operatorname{dualp}\left(E_{j}\right)=\sum_{j=1}^{n} \operatorname{dual}\left(p\left(A / E_{j}\right) p\left(E_{j}\right)\right) .
$$

\section{Case Study}

Let us consider a "classical” task of assessing chances of the presence of oil on the site given that the prior evaluations of these chances and evaluations of conditional probabilities of the results of seismic exploration of the site are assigned. We have these initial data.

A set of random events (",states of nature") $A=\left\{a_{1}, a_{2}\right\}$ where event $a_{1}$ corresponds to the actual presence of oil on the site, but event $a_{2}$ corresponds to real absence of oil on the site. Let us call events $a_{1}$ and $a_{2}$ "geological events". Let us assume that based on the expert evaluation, the following interval values of probabilities of occurrence of these events are assigned: $p\left(a_{1}\right)=[0.50,0.70], p\left(a_{2}\right)=[0.30,0.50]$.

Assume that a manager of an oil mining company has made a decision to undertake seismic exploration of the site to re-evaluate the prior values of probabilities $p\left(a_{1}\right)$ and $p\left(a_{2}\right)$.

Let us denote a set of random events, outcomes of seismic exploration as $B=\left\{b_{1}, b_{2}\right\}$ where $b_{1}$ is an outcome that indicates the presence of oil on the site but $b_{2}$ is an outcome indicating the absence of oil on the site. Let us call events $b_{1}$ and $b_{2}$ seismic events. 
The specifics of a seismic exploration is that it can both precisely confirm real presence or absence of oil on a site, and produce erroneous results, i.e., to show the presence of oil when it is missing in reality or to show the absence of oil when it is really present. Let us introduce this system of denotations:

$b_{1} / a_{1}$ - seismic exploration has confirmed real presence of oil on the site;

$b_{2} / a_{1}$ - seismic exploration has erroneously indicated the lack of oil on the site, though in reality oil is present;

$b_{1} / a_{2}$ - seismic exploration has erroneously indicated the presence of oil on the site, though in reality there is no oil on the site;

$b_{2} / a_{2}$ - seismic exploration has confirmed that there is no oil on the site.

Let there be set these interval values of conditional probabilities:

$$
\begin{aligned}
& p\left(b_{1} / a_{1}\right)=[0.70,0.90], \quad p\left(b_{1} / a_{2}\right)=[0.10,0.30] \\
& p\left(b_{2} / a_{1}\right)=[0.10,0.30], \quad p\left(b_{2} / a_{2}\right)=[0.70,0.90] .
\end{aligned}
$$

Initial state of information is shown in Figure 1 as a decision tree.

Geological events Seismic events

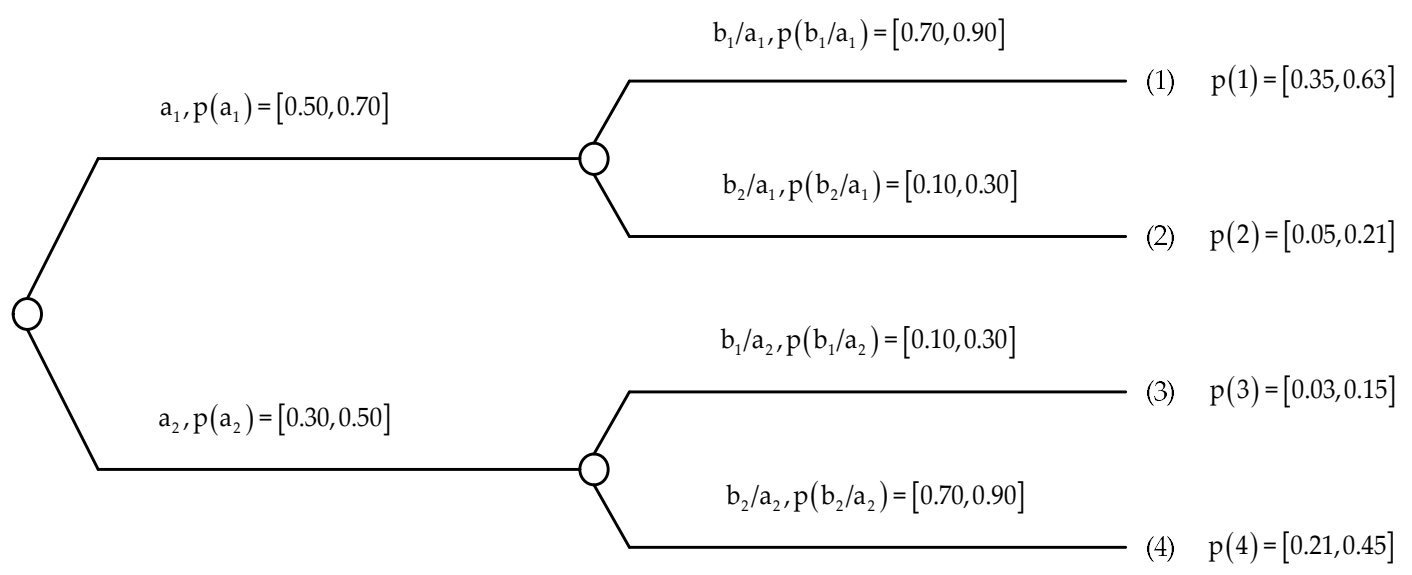

Figure 1. Decision tree depicting initial state of information.

Actually, we are not interested in conditional probabilities $p\left(b_{j} / a_{i}\right), i, j=1,2$, depicting results of seismic exploration depending on the real presence or lack of oil on the site; instead, we are interested in conditional probabilities $p\left(a_{i} / b_{j}\right), i, j=1,2$ that show the presence or lack of oil on the site depending on the results of seismic exploration. As in any practical use of interval probabilities, it is necessary to operate only with reachable probability intervals. It is easy to verify that in the present example, initial probability intervals are reachable. Our task in this example is to calculate the posterior probabilities $p\left(a_{i} / b_{j}\right), i, j=1,2$, based on all available information. 
Let us calculate values of outcome probabilities (scenarios) using the data shown on the decision tree in Figure 1.

$$
\begin{aligned}
& p(1)=p\left(a_{1}\right) p\left(b_{1} / a_{1}\right)=[0.50,0.70]^{*}[0.70,0.90]=[0.35,0.63] \\
& p(2)=p\left(a_{1}\right) p\left(b_{2} / a_{1}\right)=[0.50,0.70] *[0.10,0.30]=[0.05,0.21] \\
& p(3)=p\left(a_{2}\right) p\left(b_{1} / a_{2}\right)=[0.30,0.50] *[0.10,0.30]=[0.03,0.15] \\
& p(4)=p\left(a_{2}\right) p\left(b_{2} / a_{2}\right)=[0.30,0.50] *[0.70,0.90]=[0.21,0.45]
\end{aligned}
$$

The values of these probabilities are depicted at the ends of the branches of the decision tree in Figure 1.

The requested posterior probabilities of geological events can be calculated using Bayes' formula directly. In the above example, however, we can make calculations by a simpler technique based on Bayes' formula.

Event $b_{1}$ can occur together with $a_{1}$ (outcome (1)) and together with event $a_{2}$ (outcome (3)). Hence, the total probability of event $b_{1}$ can be calculated as

$$
\mathrm{p}^{\prime}\left(\mathrm{b}_{1}\right)=\mathrm{p}(1)+\mathrm{p}(3)=[0.40,0.56]+[0.06,0.10]=[0.46,0.56]
$$

Event $b_{2}$ can occur together with event $a_{1}$ (outcome (2)) and event $a_{2}$ (outcome (4)). Therefore,

$$
\mathrm{p}^{\prime}\left(\mathrm{b}_{2}\right)=\mathrm{p}(2)+\mathrm{p}(4)=[0.10,0.14]+[0.24,0.40]=[0.34,0.54]
$$

Now we have all necessary information for calculating the requested values of the posterior probabilities. For that purpose, we will use expressions $(12, a, b)$. In those expressions, to calculate the denominators, it is necessary to use the boundary values of the total probabilities $p^{\prime}\left(b_{1}\right)$ and $p^{\prime}\left(b_{2}\right)$. Since expressions $(12, a, b)$ are a special case of general expression $(10)$, it is necessary to use inverted values of $p^{\prime}\left(b_{1}\right)$ and $p^{\prime}\left(b_{2}\right)$, namely

$$
\begin{aligned}
& \mathrm{p}^{\prime \prime}\left(\mathrm{b}_{1}\right)=\operatorname{dualp}^{\prime}\left(\mathrm{b}_{1}\right)=[0.66,0.46] \\
& \mathrm{p}^{\prime \prime}\left(\mathrm{b}_{2}\right)=\operatorname{dualp}^{\prime}\left(\mathrm{b}_{2}\right)=[0.54,0.34]
\end{aligned}
$$

To calculate the required posterior interval probabilities, it is necessary to divide the outcome probability values $p(i), \quad i=1,2,3,4$, by the corresponding values $p^{\prime \prime}\left(b_{i}\right), i=1,2$. We have

$$
\begin{aligned}
& \mathrm{p}\left(\mathrm{a}_{1} / \mathrm{b}_{1}\right)=\frac{\mathrm{p}(1)}{\mathrm{p}^{\prime \prime}\left(\mathrm{b}_{1}\right)}=\frac{[0.40,0.46]}{[0.66,0.46]}=\left[\frac{0.40}{0.46}, \frac{0.56}{0.66}\right] \approx[0.85,0.87] \\
& \mathrm{p}\left(\mathrm{a}_{1} / \mathrm{b}_{2}\right)=\frac{\mathrm{p}(2)}{\mathrm{p}^{\prime \prime}\left(\mathrm{b}_{2}\right)}=\frac{[0.10,0.14]}{[0.54,0.34]}=\left[\frac{0.10}{0.34}, \frac{0.14}{0.54}\right] \approx[0.29,0.26] \\
& \mathrm{p}\left(\mathrm{a}_{2} / \mathrm{b}_{1}\right)=\frac{\mathrm{p}(3)}{\mathrm{p}^{\prime \prime}\left(\mathrm{b}_{1}\right)}=\frac{[0.06,0.10]}{[0.66,0.46]}=\left[\frac{0.06}{0.46}, \frac{0.10}{0.66}\right] \approx[0.13,0.15]
\end{aligned}
$$




$$
\mathrm{p}\left(\mathrm{a}_{2} / \mathrm{b}_{2}\right)=\frac{\mathrm{p}(4)}{\mathrm{p}^{\prime \prime}\left(\mathrm{b}_{2}\right)}=\frac{[0.24,0.40]}{[0.54,0.34]}=\left[\frac{0.24}{0.34}, \frac{0.40}{0.54}\right] \approx[0.71,0.74]
$$

As a result of calculation, we obtained improper resulting interval $\mathrm{p}\left(\mathrm{a}_{1} / \mathrm{b}_{2}\right)=[0.29,0.26]$.

This is due to the peculiarities of operating with generalised probability intervals.To get the correct result, we simply invert this inactive probability interval. As a result, we have

$$
\mathrm{p}\left(\mathrm{a}_{1} / \mathrm{b}_{1}\right)=[0.85,0.87], \mathrm{p}\left(\mathrm{a}_{1} / \mathrm{b}_{2}\right)=[0.26,0.29], \mathrm{p}\left(\mathrm{a}_{2} / \mathrm{b}_{1}\right)=[0.13,0.15], \mathrm{p}\left(\mathrm{a}_{2} / \mathrm{b}_{2}\right)=[0.71,0.74] \text {. }
$$

It is easy to verify that the resulting intervals are valid probability intervals. The target state of information in the form of a decision tree is presented in Figure 2.

Seismic events Geological events

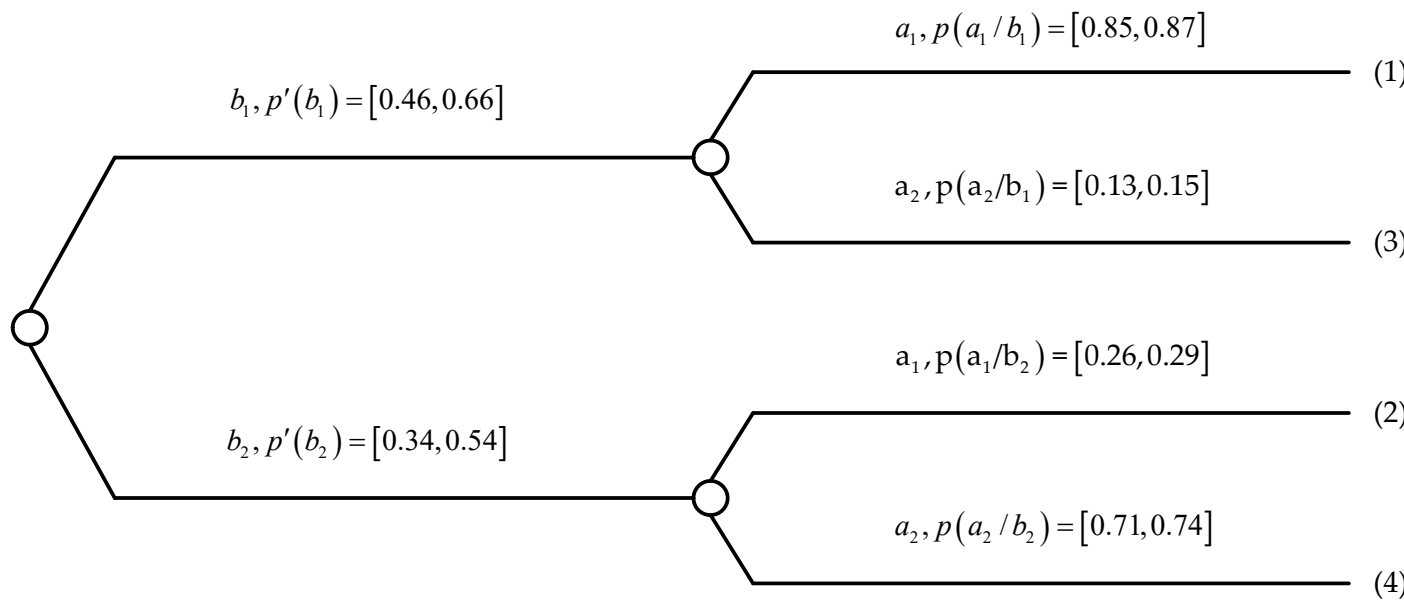

Figure 2. A probability tree that displays the target state of information.

Note that on this probability tree, the numbering of outcomes corresponds to the numbering of outcomes on the probability tree in Figure 1. The probabilities of the respective outcomes in both figures are the same.

\section{Algorithms for Finding Permissible Values of Probabilities on Sets of Their Interval Values}

Let us introduce the concept of consistent probability intervals. Let us assume that a set of permissible intervals $\mathrm{L}=\left\{\mathrm{l}_{\mathrm{i}}, \mathrm{u}_{\mathrm{i}} / \mathrm{i}=1, \ldots, \mathrm{n}\right\}$ is set, and for each interval, its average point $c_{i}$ is assigned. Let us call these intervals consistent if these conditions hold: (1) the width of all intervals is the same, $\left[l_{i}, u_{i}\right]=$ const,$\forall i$, and (2) $\sum_{i=1}^{n} c_{i}=1$.

The concept of consistent intervals does not seem to be significantly limiting. It can be assumed that the expert first sets the point values of the estimated probabilities with the condition that the sum of the probabilities is 1 ; then he models the uncertainties of his estimates, setting the same intervals for all initial estimates. 
In practical problems of various kinds, sometimes it becomes necessary to analyse the set of determinate probability values fixed on the set of their interval values. As an illustrative example, we can cite the problem of analysing the probabilistic sensitivity of decisions if the probabilities of outcomes of alternative decisions are presented in interval form. By fixing these or those values of these probabilities of interest to us at given intervals of their possible values, we can evaluate how robustly the optimal solution is regarding the possible ranges of changes in relevant probabilities.

In this regard, the following problem arises: there are $n$ interval consistent values of the probabilities, and we need to choose one valid determinate probability value, $p_{i}, i=1, \ldots, n$, from each interval. By permissible, we mean probability values that satisfy the basic requirement of the classical theory of probability: for a complete group of random events, $\sum_{\mathrm{i}=1}^{\mathrm{n}} \mathrm{p}_{\mathrm{i}}=1$.

In order to correctly select the permissible probability values of interest to us on the set of their interval values, it is proposed to use the following algorithm [2]. We consider the variants of the algorithms for the cases of two, three and four consistent interval probability values.

Let there be given two consistent interval probability values: $p_{1}=\left[1_{1}, u_{1}\right]$ and $p_{2}=\left[1_{2}, u_{2}\right]$. For clarity, two such conditional intervals are graphically presented in Figure 3.

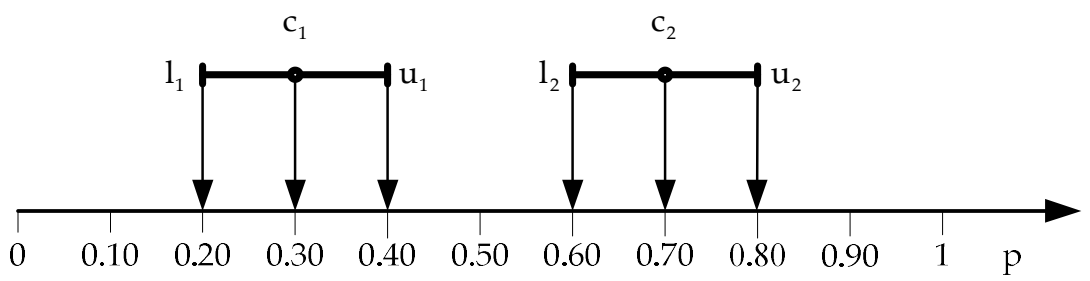

Figure 3. Graphic representation of two conditional consistent probability intervals.

In this figure, the centres of the corresponding intervals are denoted by $c_{1}$ and $c_{2}$. For these intervals to be consistent, they must satisfy the following requirements:(1) $c_{1}+c_{2}=1$; (2) $\mathrm{u}_{1}-\mathrm{l}_{1}=\mathrm{u}_{2}-\mathrm{l}_{2}$.

Let there be set two consistent probability intervals $p_{1}=\left[l_{1}, u_{1}\right]$ and $p_{2}=\left[l_{2}, u_{2}\right]$ and let an arbitrary deterministic value $\mathrm{p}_{1} \in\left[\mathrm{l}_{1}, \mathrm{u}_{1}\right]$ be selected on the first interval. Then the corresponding to it permissible probability value $\mathrm{p}_{2} \in\left[\mathrm{l}_{2}, \mathrm{u}_{2}\right]$ is determined as $\mathrm{p}_{2}=1-\mathrm{p}_{1}$.

1. Three consistent probability intervals are given: $p_{1}=\left[1_{1}, u_{1}\right], p_{2}=\left[1_{2}, u_{2}\right]$ and $p_{3}=\left[1_{3}, u_{3}\right]$. For clarity, three such conditional intervals are graphically presented in Figure 4.

2. For these intervals to be consistent, they must satisfy the following requirements: (1) $\mathrm{c}_{1}+\mathrm{c}_{2}+\mathrm{c}_{3}=1 ;(2) \mathrm{u}_{1}-\mathrm{l}_{1}=\mathrm{u}_{2}-\mathrm{l}_{2}=\mathrm{u}_{3}-\mathrm{l}_{3}$.

3. The algorithm for determining permissible probability values in this case works as follows. The fixed probability value $\mathrm{p}_{1} \in\left[\mathrm{l}_{1}, \mathrm{u}_{1}\right]$ is set. Depending on the actual value of $\mathrm{p}_{1}$, the following options are possible for determining the intervals in which permissible values of probabilities $\mathrm{p}_{2}$ and $\mathrm{p}_{3}$ are located: 


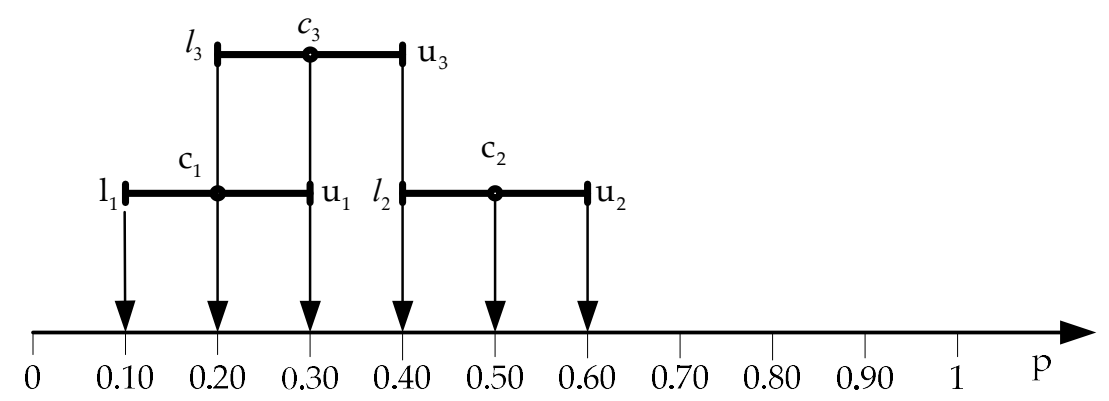

Figure 4. Graphic representation of three conditional consistent probability intervals.

(1) If $\mathrm{p}_{1}=\mathrm{l}_{1}$, then $\mathrm{p}_{2} \in\left[\mathrm{c}_{2}, \mathrm{u}_{2}\right], \mathrm{p}_{3} \in\left[\mathrm{u}_{3}, \mathrm{c}_{3}\right]$;

(2) If $\mathrm{p}_{1}=\mathrm{l}_{1}+\gamma, 0<\gamma<\mathrm{c}_{1}-\mathrm{l}_{1}$, then $\mathrm{p}_{2} \in\left[\mathrm{c}_{2}-\gamma, \mathrm{u}_{2}\right], \mathrm{p}_{3} \in\left[\mathrm{u}_{3}, \mathrm{c}_{3}-\gamma\right]$;

(3) If $\mathrm{p}_{1}=\mathrm{c}_{1}$, then $\mathrm{p}_{2} \in\left[\mathrm{l}_{2}, \mathrm{u}_{2}\right], \mathrm{p}_{3} \in\left[\mathrm{u}_{3}, \mathrm{l}_{3}\right]$;

(4) If $\mathrm{p}_{1}=\mathrm{c}_{1}+\delta, 0<\delta<\mathrm{u}_{2}-\mathrm{c}_{1}$, then $\mathrm{p}_{2} \in\left[\mathrm{l}_{2}, \mathrm{u}_{2}-\delta\right], \mathrm{p}_{3} \in\left[\mathrm{u}_{3}-\delta, 1_{3}\right]$;

(5) If $\mathrm{p}_{1}=\mathrm{u}_{1}$, then $\mathrm{p}_{2} \in\left[\mathrm{l}_{2}, \mathrm{c}_{2}\right], \mathrm{p}_{3} \in\left[\mathrm{c}_{3}, 1_{3}\right]$.

Let there be given four consistent interval probability values: $\mathrm{p}_{1}=\left[\mathrm{l}_{1}, \mathrm{u}_{1}\right], \mathrm{p}_{2}=\left[\mathrm{l}_{2}, \mathrm{u}_{2}\right], \mathrm{p}_{3}=\left[\mathrm{l}_{3}, \mathrm{u}_{3}\right]$ and $\mathrm{p}_{4}=\left[\mathrm{l}_{4}, \mathrm{u}_{4}\right]$. Consistency in this case means fulfilling the following requirements:

(1) $\mathrm{c}_{1}+\mathrm{c}_{2}+\mathrm{c}_{3}+\mathrm{c}_{4}=1$; (2) $\mathrm{u}_{1}-\mathrm{l}_{1}=\mathrm{u}_{2}-\mathrm{l}_{2}=\mathrm{u}_{3}-\mathrm{l}_{3}=\mathrm{u}_{4}-\mathrm{l}_{4}$.

For clarity, four such conditional intervals are shown graphically in Figure 5.

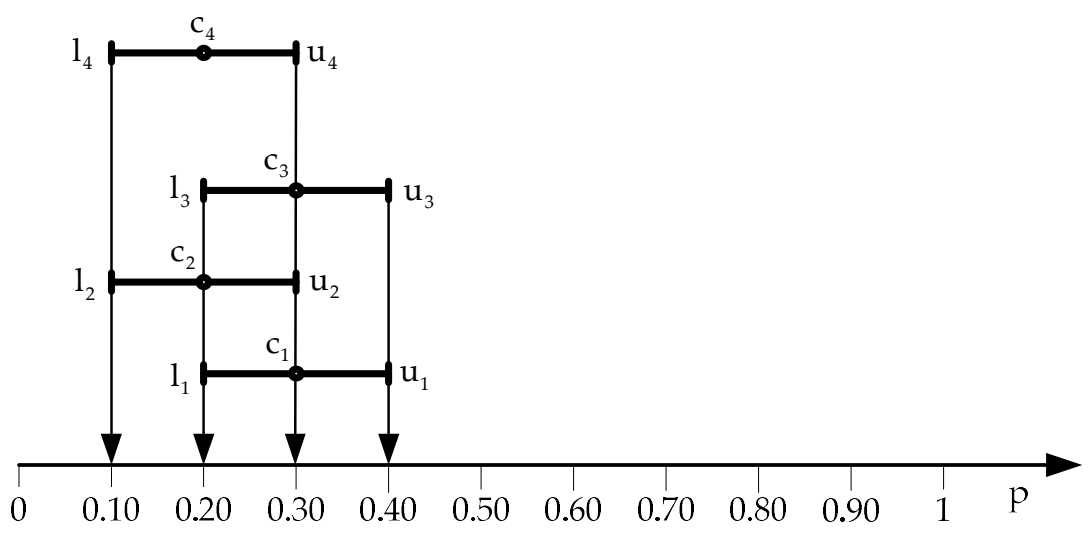

Figure 5. Graphic representation of four conditional consistent probability intervals.

In this case, the algorithm for determining permissible probabilities $p_{i}, \mathrm{i}=1,2,3,4$ works as follows. Two probabilities of interest to us, $p_{1}$ and $p_{2}$ are fixed, and their sum, $p_{1}+p_{2}$ is calculated. Depending on the value of this sum, the intervals of permissible probability values $p_{3}$ and $\mathrm{p}_{4}$ are determined as follows: 
(1) If $p_{1}+p_{2}=l_{1}+1_{2}$, then $p_{3}=u_{3}, p_{4}=u_{4}$.

(2) $l_{1}+l_{2}<p_{1}+p_{2}<c_{1}+c_{2}$. Let us denote the difference $\left(p_{1}+p_{2}\right)-\left(l_{1}+l_{2}\right)$ by $\gamma$. Then

$\mathrm{p}_{3} \in\left[\mathrm{u}_{3}-\gamma, \mathrm{u}_{3}\right]$ and $\mathrm{p}_{4} \in\left[\mathrm{u}_{4}, \mathrm{u}_{4}-\gamma\right]$

(3) If $\mathrm{p}_{1}+\mathrm{p}_{2}=\mathrm{c}_{1}+\mathrm{c}_{2}$, then $\mathrm{p}_{3} \in\left[\mathrm{l}_{3}, \mathrm{u}_{3}\right], \mathrm{p}_{4} \in\left[\mathrm{u}_{4}, \mathrm{1}_{4}\right]$.

(4) $\mathrm{c}_{1}+\mathrm{c}_{2}<\mathrm{p}_{1}+\mathrm{p}_{2}<\mathrm{u}_{1}+\mathrm{u}_{2}$. Let us denote by $\delta$ the difference $\left(\mathrm{p}_{1}+\mathrm{p}_{2}\right)-\left(\mathrm{c}_{1}+\mathrm{c}_{2}\right)$. Then

$\mathrm{p}_{3} \in\left[\mathrm{l}_{3}, \mathrm{u}_{3}-\delta\right]$ and $\mathrm{p}_{4} \in\left[\mathrm{u}_{4}-\delta, 1_{4}\right]$

(5) If $p_{1}+p_{2}=u_{1}+u_{2}$, then $p_{3}=l_{3}, p_{4}=1_{4}$.

The algorithm can be extended to a larger number of initial probability intervals. Let the number of initial probability intervals be equal to $n>4$. Then, at first, $n-2$ acceptable probabilities must be selected and fixed. The values of the remaining permissible probabilities are determined as for the case of four probability intervals, taking into account the sum of the probabilities given to the first $\mathrm{n}-2$ intervals.

\section{Findings and Conclusions ${ }^{\circ}$}

Interval probability estimates model expert uncertainties regarding estimated probabilities. To operate with such uncertain probabilistic estimates, a special mathematical apparatus is required. In this paper, we consider an approach to calculating posterior conditional probabilities based on the concept of generalized intervals $[8,9]$. This approach seems simpler and more intuitive than those presented in [4-7]. The paper presents an algorithm for determining the sets of permissible probabilistic estimates on the set of their interval values.

The use of interval probabilistic estimates seems to be a necessary measure in cases when sufficient statistical data are missing and expert uncertainties regarding the required probability estimates are present.

Interval probabilities are widespread in various scientific and practical fields. In [9], an approach was proposed to assess the reliability of systems with imprecise probability values in the form of generalized intervals. This work can be considered as a continuation of [8] and an attempt to apply the theoretical approach proposed in both works to the solution of practical engineering problems. A good survey on applications of imprecise probabilities in various engineering fields can be found in [11].

The concept of interval probabilities has found wide application in various areas of decision theory. In [12], the author paid great attention to the problems of obtaining and processing interval information. An original approach to the selection of optimal solutions for imprecise outcome probabilities was also proposed there.

Another approach to the choice of solutions for interval values of relevant probabilities was proposed in [13]. The author offers an imprecise hierarchical decision-making model where interval probabilities are used at the first and second levels. 
In [14], the author proposes an extension of the classical theory of utility in decision-making for the case of interval probabilities. These and other examples clearly indicate that interval probabilities are widely used in various fields.

Author Contributions: Conceptualization, O.U-R; methodology, O.U-R; validation, O.U-R and G.K; formal analysis, O.U-R and G.K.; investigation, O.U-R and G.K.; resources, G.K.; data curation, G.K.; writing, - original draft preparation, O.U-R and G.K, writing-review and editing O.U-R., supervision O.U-R. All authors have read and agreed to the published version of the manuscript.

Funding: This research received no external funding.

Conflicts of Interest: The authors declare no conflict of interest.

\section{References}

1. Borisov, A.N., Uzhga-Rebrov, O.I. and Savchenko, K.I. Probabilistic reasoning in intelligent systems, Riga, Latvia, 20020, 216 p. (In Russian)

2. Uzhga-Rebrov, O.I. (2019). Estimation, analysis and propagation of uncertainties. SIARAdrukātava, Rezekne, Latvia, 2019, 582 p. (In Russian)

3. De Campos, L.M., Huete, J.F. and Moral, S. (1994). Probability Intervals: A Tool for Uncertain Reasoning. Int. Journal of Uncertainty, Fuzziness and Knowledge-Based Systems, 1994, Vol. 2, No. 2, pp. 167 - 196.

4. Weichselberger, K. The Theory of Interval Probability as a Unifying Concept for Uncertainty. $1^{\text {st }}$ Int. Symposium on Imprecise Probabilities and Their Application, Ghent, Belgium, 29 June 2 July, 1999, 9 p.

5. Weichselberger, K. Elementare Grundbegriffe einer allgemeinen Wahrscheinlichkeitsrechnung I - Intervallwahrscheinlichkeitn als umfassendes Konzept. In cooperation with T. Augustin and Wallner A., 2001, Heidelberg, Physica.

6. Weichselberger, K. and Augustin, Th. On the Symbiosis of Two Concepts of Conditional Interval Probability. In J. Bernard, T. Seidenfeld, and M. Zaffalon (Eds), ISIPTA'03, 2003, Waterloo, Carleton Scientific,pp. $608-630$.

7. Weichselberger, K. The Logical Concept of Probability and Statistical Inference.4 ${ }^{\text {th }}$ Int. Symposium on Imprecise Probabilities and Their Applications. Pittsburg,Pennsylvania, 2005, 10 p.

8. Wang, Y. Imprecise Probabilities with a Generalized Interval Form. Proc. 3rd Int. Workshop on Reliability Engineering, 2008, 15 p.

9. Wang, Y. Imprecise Probabilities Based on Generalized Intervals for System Reliability Assessment. Int. Journal Reliability and Safety, 2010, Vol. 4, No. 4, pp.319-342.

10. Kaucher, E. Interval analysis in the extended interval space IR. Computing Supplement, 1980,2, pp. $33-49$.

11. Beer, M., Ferson, S. and Kreinovich, V. Imprecise probabilities in engineering analyses. Mechanical Systems and Signal Processing, 2013, Vol. 37, Issue 1-2, pp. 4-29.

12. Coolen, F.P.A. Statistical modelling of expert opinions using imprecise probabilities.Eindhowen: Technische Universteit Eindhowen, 1994, doi 10.6100/IR410210, 126 p.

13. Zeinalova, L.M. Decision making with combined states under internal uncertainty. Procedia Computer Science, 2016, Vol. 102, pp. 223 - 230.

14. Augustin, Th. Generalized basic probability assignments. Ludwig-Maximilians Universität, München, Sonderforschungsbereich, 2002, 386, Paper 276, 14 p. 